\title{
Convex Polygons are Cover-Decomposable
}

\author{
Dömötör Pálvölgyi • Géza Tóth
}

Received: 22 August 2008 / Revised: 22 December 2008 / Accepted: 22 December 2008 /

Published online: 4 March 2009

(C) Springer Science+Business Media, LLC 2009

\begin{abstract}
We show that for any open convex polygon $P$, there is a constant $k(P)$ such that any $k(P)$-fold covering of the plane with translates of $P$ can be decomposed into two coverings.
\end{abstract}

Keywords Multiple coverings $\cdot$ Decomposability $\cdot$ Wedges

\section{Introduction}

Let $\mathcal{P}=\left\{P_{i} \mid i \in I\right\}$ be a collection of planar sets. It is a $k$-fold covering if every point in the plane is contained in at least $k$ members of $\mathcal{P}$. A one-fold covering is simply called a covering.

Definition A planar set $P$ is said to be cover-decomposable if the following holds. There exists a constant $k=k(P)$ such that every $k$-fold covering of the plane with translates of $P$ can be decomposed into two coverings. J. Pach [4] proposed the problem of determining all cover-decomposable sets in 1980. For related problems and conjectures, see [2], Chap. 2.1 and [7].

D. Pálvölgyi was supported by OTKA NK 67867.

G. Tóth was supported by OTKA T 038397 and T 046246.

D. Pálvölgyi

ELTE, Budapest, Hungary

D. Pálvölgyi

EPFL, Lausanne, Switzerland

G. Tóth $(\bowtie)$

Rényi Institute, Hungarian Academy of Sciences, Budapest, Hungary

e-mail: geza@renyi.hu 
Conjecture (J. Pach) All planar convex sets are cover-decomposable.

This conjecture is verified in three special cases.

\section{Theorem A}

(i) [5] Every centrally symmetric open convex polygon is cover-decomposable.

(ii) [3] The open unit disc is cover-decomposable.

(iii) [9] Every open triangle is cover-decomposable.

In this note we verify the conjecture for open convex polygons.

\section{Theorem 1 Every open convex polygon is cover-decomposable.}

Just like in [5] and [9], we formulate and solve the problem in its dual form. That is, suppose that $P$ is a polygon of $n$ vertices and that we have a collection $\mathcal{P}=\left\{P_{i} \mid\right.$ $i \in I\}$ of translates of $P$. Let $O_{i}$ be the center of gravity of $P_{i}$. The collection $\mathcal{P}$ is a $k$-fold covering of the plane if and only if every translate of $\bar{P}$, the reflection of $P$ through the origin, contains at least $k$ points of the collection $\mathcal{O}=\left\{O_{i} \mid i \in I\right\}$.

The collection $\mathcal{P}=\left\{P_{i} \mid i \in I\right\}$ can be decomposed into two coverings if and only if the set $\mathcal{O}=\left\{O_{i} \mid i \in I\right\}$ can be colored with two colors so that every translate of $\bar{P}$ contains a point of both colors.

Divide the plane into small regions, say, squares, such that each square contains at most one vertex of any translate of $\bar{P}$. If a translate of $\bar{P}$ contains sufficiently many points of $\mathcal{O}$, then it contains many points of $\mathcal{O}$ in one of the little squares. We color the points of $\mathcal{O}$ separately in each of the squares. If we concentrate on points in just one of the little squares, then instead of translates of $\bar{P}$ we can consider translates of $n$ different wedges corresponding to the $n$ vertices of $P$.

In Sect. 2 we prove some results about coloring point sets with respect to translates of wedges. In Sect. 3 we formulate the problem precisely in the dual version and apply the results of Sect. 2 to prove Theorem 1.

\section{Preparation}

Suppose we have two halflines, $e$ and $f$, both of endpoint $O$. Then they divide the plane into two parts, $W_{1}$ and $W_{2}$, which we call wedges. A closed wedge contains its boundary, while an open wedge does not. The point $O$ where the two boundary lines meet is called the apex of the wedges. The angle of a wedge is the angle between its two boundary halflines, measured inside the wedge. That is, the sum of the angles of $W_{1}$ and $W_{2}$ is $2 \pi$. Now let $W$ be a wedge, and $X$ be a point in the plane. A translate of $W$ such that its apex is at $X$ is denoted by $W(X)$. More generally, for points $X_{1}, X_{2}, \ldots X_{k}, W\left(X_{1}, X_{2}, \ldots X_{k}\right)$ denotes the minimal translate of $W$ (for containment) whose closure contains $X_{1}, X_{2}, \ldots X_{k}$. The set of all translates of $W$ is denoted by $\operatorname{Tr}^{W}$. Let $-W$ denote the reflection of $W$ about the origin.

Definition 1 Suppose that $\mathcal{W}=\left\{W_{i} \mid i \in I\right\}$ is a collection of wedges. $\mathcal{W}$ is said to be nonconflicting or simply NC if there is a constant $k$ with the following property. 
Any finite set of points $S$ can be colored with two colors so that any translate of a wedge $W \in \mathcal{W}$ that contains at least $k$ points of $S$ contains points of both colors.

For any fixed $\mathcal{W}$ and $S$, we can and will assume without loss of generality that the points of $S$ are in general position with respect to $\mathcal{W}$, that is, they do not determine a line parallel to the boundary line of any $W \in \mathcal{W}$. Indeed, if there are two such points, slightly perturb the points. It is easy to see that every subset of the original point set that can be cut off by a translate of a $W \in \mathcal{W}$ can also be cut off the perturbed point set.

First suppose that we have only one open wedge, that is, $\mathcal{W}=\{W\}$.

\section{Lemma 1 A single wedge is NC.}

Proof Let $S$ be a finite point set and $W$ a wedge. We prove the statement with $k=3$, that is, $S$ can be colored with two colors so that any translate of $W$ that contains at least three points of $S$ contains a point of both colors. Suppose first that the angle of $W$ is at least $\pi$. Then $W$ is the union of two halfplanes, $A$ and $B$. Take the translate of $A$ (resp. $B$ ) that contains exactly two points of $S$, say, $A_{1}$ and $A_{2}$ (resp. $B_{1}$ and $B_{2}$ ). There might be coincidences between $A_{1}, A_{2}$ and $B_{1}, B_{2}$; therefore the set $\left\{A_{1}, A_{2}, B_{1}, B_{2}\right\}$ contains two, three, or four different points. However, in any case, we can color $\left\{A_{1}, A_{2}, B_{1}, B_{2}\right\}$ so that $A_{1}$ and $A_{2}$ (resp. $B_{1}$ and $B_{2}$ ) are of different colors. Now, if a translate of $W$ contains three points, it contains either $A_{1}$ and $A_{2}$, or $B_{1}$ and $B_{2}$, and we are done.

Suppose now that the angle of $W$ is less than $\pi$. We give two proofs in this case, since we will apply the ideas of both proofs later. In fact, we show that in this case the NC property holds with $k=2$. We can assume that the positive $x$-axis is in $W$ and that no two points have the same $y$-coordinate. Both of these can be achieved by an appropriate rotation. We say that $X<_{y} Y$ if the $y$-coordinate of $X$ is smaller than the $y$-coordinate of $Y$. This ordering is called the $y$-ordering. A subset $I$ of $S$ is an interval of $S$ if $\forall X<_{y} Y<_{y} Z \in S: X, Z \in I \rightarrow Y \in I$.

The boundary of $S$ with respect to $W$ is defined as $B d^{W}(S)=\{X \in P: W(X) \cap$ $S=\emptyset\}$. Note that a translate of $W$ always intersects the boundary in an interval. For each $X \in B d^{W}(S)$, the shadow of $X$ is $S h^{W}(X)=\left\{Y \in S: W(Y) \cap B d^{W}(S)=X\right\}$. Observe that $\forall X, Y \in B d^{W}(S): S h^{W}(X) \cap S h^{W}(Y)=\emptyset$.

For the first proof, color the points of the boundary alternating, according to the order $<_{y}$. For every boundary point $X$, color every point in the shadow of $X$ to the other color than $X$. Color the rest of the points arbitrarily. Any translate of $W$ that contains at least two points contains one or two boundary points. If it contains one boundary point, then the other point is in its shadow, so they have different colors. If it contains two boundary points, then they are consecutive points according to the $y$-order, so they have different colors again. This finishes the first proof.

For the second proof, for any fixed $y$, let $W(2 ; y)$ be the translate of $W$ which (1) contains at most two points of $S$, (2) its apex has $y$-coordinate $y$, and (3) its apex has minimal $x$-coordinate. It is easy to see that for any $y, W(2 ; y)$ is uniquely defined. Examine how $W(2 ; y)$ changes as $y$ runs over the real numbers. If $y$ is very small (smaller than the $y$-coordinate of the points of $S$ ), then $W(2 ; y)$ contains two 
Fig. $1 Z$ replaces $X$ in $W(2 ; y)$
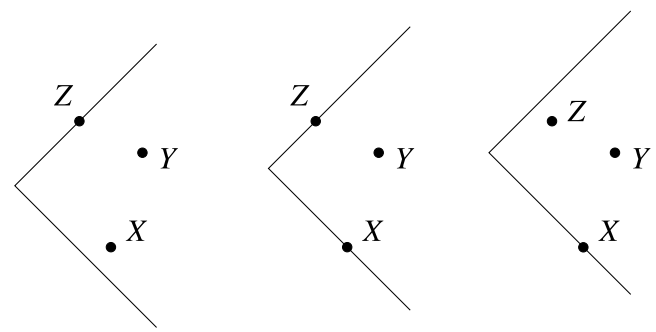

Fig. 2 Path decompositions of order two. $P_{1}=X_{1} X_{2} \ldots$, $P_{2}=Y_{1} Y_{2} \ldots$
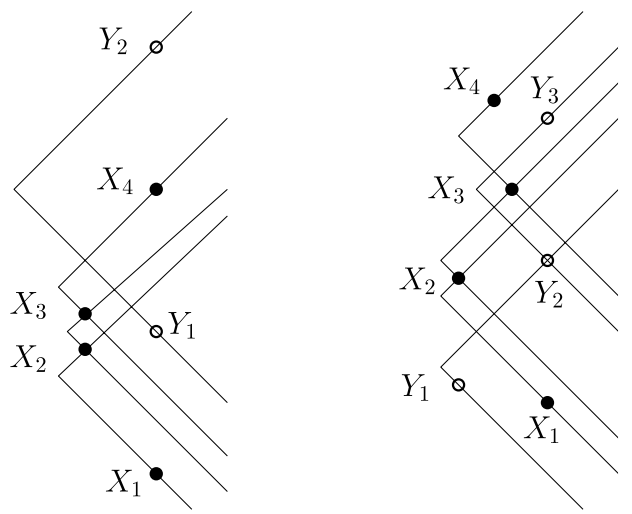

points, say $X$ and $Y$, and one more on its boundary. As we increase $y$, the apex of $W(2 ; y)$ changes continuously. How can the set $\{X, Y\}$ of two points in $W(2 ; y)$ change? For a certain value of $y$, one of them, say, $X$, moves to the boundary. At this point we have $Y$ inside and two points, $X$ and $Z$, on the boundary. If we slightly further increase $y$, then $Z$ replaces $X$, that is, $Y$ and $Z$ will be in $W(2 ; y)$ (see Fig. 1). As $y$ increases to infinity, the set $\{Z, Y\}$ could change several times, but the same way. Define a graph whose vertices are the points of $S$, and two vertices $U$ and $V$ are connected iff $V$ replaced $U$ during the procedure. We get two paths, $P_{1}$ and $P_{2}$. The pair $\left(P_{1}, P_{2}\right)$ is called the path decomposition of $S$ with respect to $W$ of order two.

In Fig. 2, two examples are given. The vertices of $P_{1}$ are $X_{1}, X_{2}, \ldots$, and the vertices of $P_{2}$ are $Y_{1}, Y_{2}, \ldots$ In both examples, the translates of $W$ are given in the positions when some point is replacing another.

Color the vertices of $P_{1}$ red, the vertices of $P_{2}$ blue. Observe that each translate of $W$ that contains at least two points contains at least one vertex of both $P_{1}$ and $P_{2}$. This completes the second proof.

Now we can turn to the case where we have translates of two wedges at the same time. We distinguish several cases according to the relative position of the two wedges $V$ and $W$.

Type 1 (Big). One of the wedges has angle at least $\pi$.

For the other cases, we can assume without loss of generality that $W$ contains the positive $x$-axis. Extend the boundary halflines of $W$ to lines. They divide the plane into four parts, Upper, Lower, Left, and Right, which is $W$ itself. See Fig. 3. 
Fig. 3 Wedge $W$

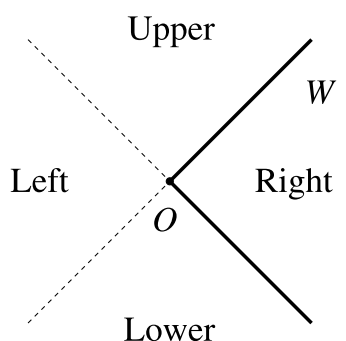

Fig. 4 Type 2 (Halfplane)
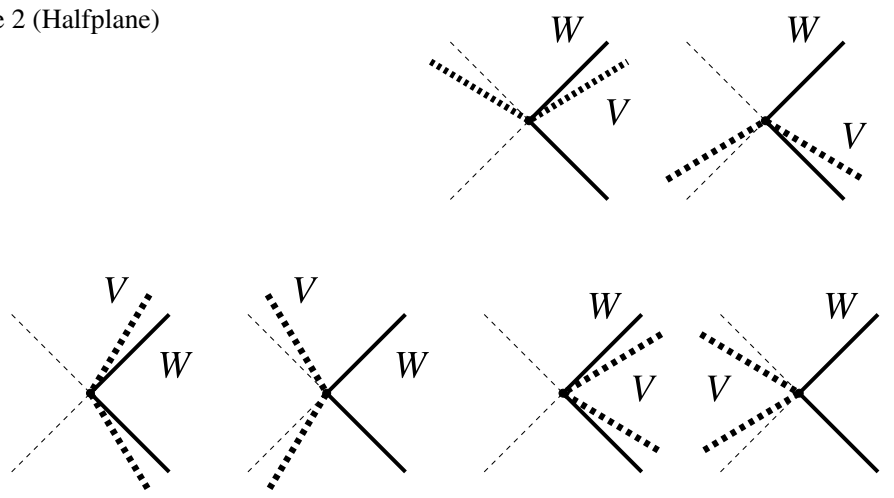

Fig. 5 Type 3 (Contain)

Fig. 6 Type 4 (Hard)

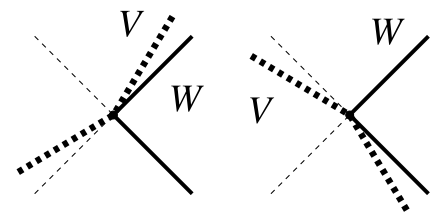

Type 2 (Halfplane). One side of $V$ is in Right, and the other one is in Left. That is, the union of the wedges cover a halfplane. See Fig. 4.

Type 3 (Contain). Either (i) one side of $V$ is in Upper, the other one is in Lower, or (ii) both sides are in Right, or (iii) both sides are in Left. See Fig. 5 .

Type 4 (Hard). One side of $V$ is in Left, and the other one is in Upper or Lower. This will be the hardest case. See Fig. 6.

Type 5 (Special). Either (i) one side of $V$ is in Right, and the other one is in Upper or Lower, or (ii) both sides are in Upper, or (iii) both sides are in Lower. That is, the union of the wedges is in an open halfplane whose boundary contains the origin, but none of them contains the other. See Fig. 7.

It is not hard to see that there are no other possibilities. Observe that two wedges corresponding to the vertices of a convex polygon cannot be of type 1 (Big) or of type 5 (Special). This will be used in the proof of Theorem 1. 

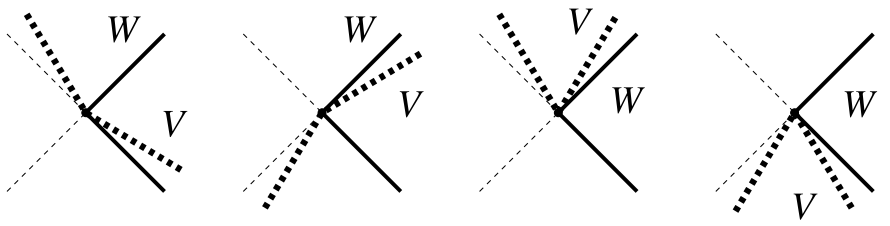

Fig. 7 Type 5 (Special)

Fig. 8

$W\left(X_{1}, X_{3}\right) \cap-W\left(X_{1}, X_{3}\right)$

$\subset V\left(X_{1}, X_{3}\right) \cap-V\left(X_{1}, X_{3}\right)$

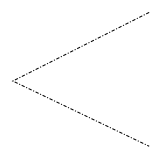

V

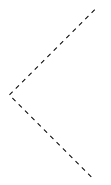

$W$

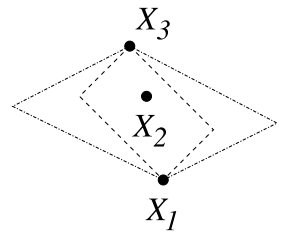

It is shown in [8] that if $\mathcal{W}=\{V, W\}$ is a set of two wedges of type 5 (Special), then $\mathcal{W}$ is not NC. In a series of lemmas we show that all other pairs are NC.

Lemma 2 Let $\mathcal{W}=\{V, W\}$ be a set of two wedges of type 3 (Contain). Then $\mathcal{W}$ is $N C$.

Suppose that $U$ is a wedge that contains the positive $x$-axis and has angle less than $\pi$. For any $k>0$ and point set $S$, let $\operatorname{Tr}_{k}^{U}(S)$ be the set of translates of $U$ that contain exactly $k$ points of $S$.

Just like in the proof of Lemma 1 , for any fixed $y$ and $k>0$, let $U(k ; y)$ be the translate of $U$ which (1) contains at most $k$ points of $S$, (2) its apex has $y$-coordinate $y$, and (3) its apex has minimal $x$-coordinate. If $y$ is very small, then $U(k ; y)$ contains $k$ points, say $X_{1}, X_{2}, \ldots, X_{k}$, and one more on its boundary. As we increase $y$, the apex of $U(k ; y)$ changes continuously. For a certain value of $y$, one of $X_{1}, X_{2}, \ldots, X_{k}$, say, $X_{1}$, moves to the boundary. At this point we have $X_{2}, \ldots, X_{k}$ inside and two points, $X_{1}$ and $X_{1}^{\prime}$, on the boundary. If we slightly further increase $y$, then $X_{1}^{\prime}$ replaces $X_{1}$, that is, $X_{1}^{\prime}$ and $X_{2}, \ldots, X_{k}$ will be in $U(k ; y)$. As $y$ increases to infinity, some other points could be replaced similarly. Define a graph whose vertices are the points of $S$ and two vertices, $X$ and $Y$, are connected iff $X$ replaced $Y$ during the procedure. We get $k$ paths $P_{1}^{U}, P_{2}^{U}, \ldots, P_{k}^{U}$. Each translate of $U$ that contains at least $k$ points of $S$ contains at least one vertex of each of $P_{1}^{U}, P_{2}^{U}, \ldots, P_{k}^{U}$. The $k$-tuple $\left(P_{1}^{U}, P_{2}^{U}, \ldots, P_{k}^{U}\right)$ is called the path decomposition of $S$ with respect to $W$ of order $k$.

Proof of Lemma 2 We can assume that $W \supset V$ or $W \supset-V$ and that $W$ contains the positive $x$-axis. Let $\left(P_{1}^{W}, P_{2}^{W}, \ldots, P_{k}^{W}\right)$ be the path decomposition of $S$ with respect to $W$ of order $k$.

Observe that any translate of $V$ intersects any $P_{i}^{W}$ in an interval of it. Indeed, if $X_{1}<y X_{2}<y X_{3} \in P_{i}^{W}$, then $X_{2} \in W\left(X_{1}, X_{3}\right) \cap-W\left(X_{1}, X_{3}\right)$, which is a subset of $V\left(X_{1}, X_{3}\right) \cap-V\left(X_{1}, X_{3}\right)$. See Fig. 8 . 
We show that we can color the points of $S$ with red and blue so that any translate of $W$ which contains at least four points and any translate of $V$ which contains at least 14 points contain points of both colors. Consider $\left(P_{1}^{W}, P_{2}^{W}, P_{3}^{W}, P_{4}^{W}\right)$, the path decomposition of $S$ with respect to $W$ of order 4 . We color $P_{1}^{W}$ and $P_{2}^{W}$ so that every $W^{\prime} \in \operatorname{Tr}_{4}^{W}(S)$ contains a blue point of them, and every $V^{\prime} \in \operatorname{Tr}_{7}^{V}\left(P_{1}^{W} \cup P_{2}^{W}\right)$ contains points of both colors. Similarly, we color $P_{3}^{W}$ and $P_{4}^{W}$ so that every $W^{\prime} \in \operatorname{Tr}_{4}^{W}(S)$ contains a red point of them, and every $V^{\prime} \in \operatorname{Tr}_{7}^{V}\left(P_{3}^{W} \cup P_{4}^{W}\right)$ contains points of both colors. Finally, we color the rest of the points $R=S \backslash\left(P_{1}^{W} \cup P_{2}^{W} \cup P_{3}^{W} \cup P_{4}^{W}\right)$ so that every $V^{\prime} \in \operatorname{Tr}_{2}^{V}(R)$ contains points of both colors.

Recall that for any $W^{\prime} \in \operatorname{Tr}_{4}^{W}(S),\left|W^{\prime} \cap P_{1}^{W}\right|=\left|W^{\prime} \cap P_{2}^{W}\right|=\left|W^{\prime} \cap P_{3}^{W}\right|=\mid W^{\prime} \cap$ $P_{4}^{W} \mid=1$. For any $X \in P_{1}^{W}$ and $Y \in P_{2}^{W}$, if there is a $W^{\prime} \in \operatorname{Tr}_{4}^{W}(S)$ with $W^{\prime} \cap P_{1}^{W}=$ $\{X\}$ and $W^{\prime} \cap P_{2}^{W}=\{Y\}$, then we say that $X$ and $Y$ are friends. If $X$ (resp. $Y$ ) has only one friend $Y$ (resp. $X$ ), then we call it a fan of $Y$ (resp. of $X$ ). If $X$ or $Y$ has at least one fan, then we say that it is a star. The points that are neither fans nor stars are called regular.

For an example, see Fig. 2. On the left figure, $Y_{1}$ is a star, its fans are $X_{2}$ and $X_{3}$, and the other points are regular. On the right, $Y_{2}$ is a star, its fan is $X_{2}$, and the other points are regular.

Suppose first that all points of $P_{1}^{W}$ and $P_{2}^{W}$ are regular. Color every third point of $P_{1}^{W}$ red and the others blue. In $P_{2}^{W}$, color the friends of the red points blue, and color the rest of the points of $P_{2}^{W}$ (every third) red. For any $W^{\prime} \in \operatorname{Tr}_{4}^{W}, W^{\prime} \cap P_{1}^{W}$ and $W^{\prime} \cap P_{2}^{W}$ are friends; therefore, at least one of them is blue. On the other hand, any $V^{\prime} \in \operatorname{Tr}_{7}^{V}\left(P_{1}^{W} \cup P_{2}^{W}\right)$ contains three consecutive points of $P_{1}^{W}$ or $P_{2}^{W}$, and they have both colors.

Suppose now that not all the points of $P_{1}^{W}$ and $P_{2}^{W}$ are regular. Color all stars blue. The first and last friend of a star, in the $y$-ordering, is either a star or a regular vertex, and the others are fans. Color the friends of each star alternatingly, according to the $y$-ordering, starting with blue, except the last two friends; color the last one blue, the previous one red. The so far uncolored regular points of $P_{1}^{W}$ and $P_{2}^{W}$ form pairs of intervals. We color each such pair of intervals the same way as we did in the all-regular case, coloring the first point of each pair of intervals red. See Fig. 9.

Clearly, if $W^{\prime} \in \operatorname{Tr}_{4}^{W}$, then it contains at least one blue point of $P_{1}^{W} \cup P_{2}^{W}$. If $V^{\prime} \in \operatorname{Tr}_{7}^{V}\left(P_{1}^{W} \cup P_{2}^{W}\right)$, then it contains four consecutive points of $P_{1}^{W}$ or $P_{2}^{W}$, say,

Fig. 9 Two examples of coloring of $P_{1}^{W} \cup P_{2}^{W}$. Friends are connected by edges
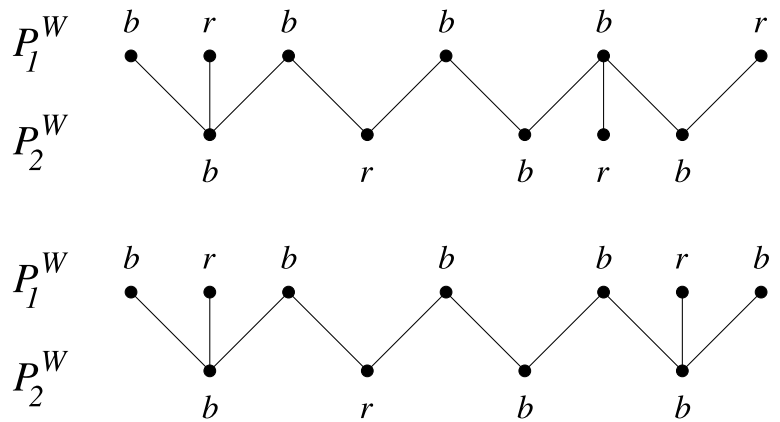
$X_{1}, X_{2}, X_{3}, X_{4}$, in $P_{1}^{W}$. If $X<_{y} Y<_{y} Z \in P_{1}^{W} \cap V^{\prime}$ and $Y$ is a star, then $V^{\prime}$ must contain all fans of $Y$ as well. Indeed, the fans of $Y$ are in $W(X, Z) \backslash(W(X) \cup W(Z))$, and by our earlier observations, this is in $V(X, Z) \subset V^{\prime}$. So, if either $X_{2}$ or $X_{3}$ is a star, then $V^{\prime}$ contains a red point, since every star has a red fan. Since the star itself is blue, we are done in this case. If $X_{1}, X_{2}, X_{3}, X_{4}$ contains three consecutive regular vertices, then we are done again, by the coloring rule for the regular intervals. So we are left with the case where $X_{1}$ and $X_{4}$ are stars, and $X_{2}$ and $X_{3}$ are regular. However, in this case, $V^{\prime}$ also contains the common friend $Y$ of $X_{2}$ and $X_{3}$ in $P_{2}^{W}$, which is also a regular vertex. By the coloring rule for the regular intervals, one of $Y, X_{2}$, and $X_{3}$ is red, the other two are blue, and so we are done.

For $P_{3}^{W} \cup P_{4}^{W}$, we use the same coloring rule as for $P_{1}^{W} \cup P_{2}^{W}$, but we switch the roles of the colors. So any $W^{\prime} \in \operatorname{Tr}_{4}^{W}$ contains at least one red point of $P_{3}^{W} \cup P_{4}^{W}$, and any $V^{\prime} \in \operatorname{Tr}_{7}^{V}\left(P_{3}^{W} \cup P_{4}^{W}\right)$ contains both colors.

Finally, we have to color the rest of the points $R=S \backslash\left(P_{1}^{W} \cup P_{2}^{W} \cup P_{3}^{W} \cup P_{4}^{W}\right)$ so that every $V^{\prime} \in \operatorname{Tr}_{2}^{V}(R)$ contains points of both colors. This can be achieved by the first proof of Lemma 1 .

Now any $W^{\prime} \in \operatorname{Tr}_{4}^{W}$ contains at least one blue and at least one red point. If $V^{\prime} \in$ $\operatorname{Tr}_{14}^{V}$, then either it contains at least two points of $R=P \backslash\left(P_{1}^{W} \cup P_{2}^{W} \cup P_{3}^{W} \cup P_{4}^{W}\right)$, or at least seven points of $P_{1}^{W} \cup P_{2}^{W}$, or at least seven points of $P_{3}^{W} \cup P_{4}^{W}$, and in all cases it contains points of both colors. This completes the proof of Lemma 2.

Definition 2 Suppose that $\mathcal{W}=\{V, W\}$ is a pair of wedges. $\mathcal{W}$ is said to be asymmetric nonconflicting or simply ANC if there is a constant $k$ with the following property. Any finite set of points $S$ can be colored with red and blue so that any translate of $V$ that contains at least $k$ points of $S$ contains a red point, and any translate of $W$ that contains at least $k$ points of $S$ contains a blue point.

The next technical result allows us to simplify all following proofs.

Lemma 3 If a pair of wedges is not of type Special and is ANC, then it is also NC.

Proof We can assume without loss of generality that $V$ contains the positive $x$-axis and that $W$ contains either the positive or negative $x$-axis. Suppose that $\{V, W\}$ is ANC, let $k>0$ be arbitrary, and let $S$ be a set of points. First we color $B d^{V}(S)$. Let $U$ be a wedge that also contains the positive $x$-axis but has a very small angle. Then translates of $V$ and translates of $U$ both intersect $B d^{V}(S)$ in its intervals. Clearly, the pair $\{U, W\}$ is of type Contain; therefore, by Lemma 2, we can color $B d^{V}(S)$ so that any translate of $W, W^{\prime} \in \operatorname{Tr}_{4}^{W}\left(B d^{V}(S)\right)$ and any translate of $U, U^{\prime} \in \operatorname{Tr}_{14}^{U}\left(B d^{V}(S)\right)$ contain both colors. However, then any translate of $V, V^{\prime} \in \operatorname{Tr}_{14}^{V}\left(B d^{V}(S)\right)$ contains both colors as well.

Now we have to color $S \backslash B d^{V}(S)$. We divide it into three parts as follows:

$$
\begin{aligned}
& S_{\mathrm{b}}=\left\{X \in S \backslash B d^{V}(S) \mid \forall Y \in V(X) \cap B d^{V}(S), Y \text { is blue }\right\}, \\
& S_{\mathrm{r}}=\left\{X \in S \backslash B d^{V}(S) \mid \forall Y \in V(X) \cap B d^{V}(S), Y \text { is red }\right\}, \\
& S_{0}=S \backslash\left(B d^{V}(S) \cup S_{\mathrm{b}} \cup S_{\mathrm{r}}\right) .
\end{aligned}
$$


Any translate $V^{\prime} \in \operatorname{Tr}^{V}$ that intersects $S_{\mathrm{b}}$ in at least one point must contain at least one blue point from $B d^{V}(S)$, so we only have to make sure that it contains a red point too. Similarly, any $V^{\prime} \in \operatorname{Tr}^{V}$ that intersects $S_{\mathrm{r}}$ in at least one point must contain a red point, and any $V^{\prime} \in \operatorname{Tr}^{V}$ that intersects $S_{0}$ must contain points of both colors.

Thus, we can simply color $S_{0}$ so that any $W^{\prime} \in \operatorname{Tr}_{2}^{W}\left(S_{0}\right)$ contains both colors, which can be done by Lemma 1 .

With $S_{\mathrm{b}}$ and $S_{\mathrm{r}}$, respectively, we proceed exactly in the same way as we did with $S$ itself, but now we change the roles of $V$ and $W$. We get the (still uncolored) subsets $S_{\mathrm{b}, \mathrm{b}}, S_{\mathrm{b}, \mathrm{r}}, S_{\mathrm{b}, 0}, S_{\mathrm{r}, \mathrm{b}}, S_{\mathrm{r}, \mathrm{r}}, S_{\mathrm{r}, 0}$ with the following properties:

- Any translate $V^{\prime} \in \operatorname{Tr}^{V}$ or $W^{\prime} \in \operatorname{Tr}^{W}$ that intersects $S_{\mathrm{b}, \mathrm{b}}$ (resp. $S_{\mathrm{r}, \mathrm{r}}$ ) in at least one point must contain at least one blue (resp. red) point.

- Any translate $V^{\prime} \in \operatorname{Tr}^{V}$ that intersects $S_{\mathrm{b}, \mathrm{r}}$ (resp. $S_{\mathrm{r}, \mathrm{b}}$ ) contains a blue (resp. red) point, and any translate $W^{\prime} \in \operatorname{Tr}^{W}$ that intersects $S_{\mathrm{b}, \mathrm{r}}$ (resp. $S_{\mathrm{r}, \mathrm{b}}$ ) contains a red (resp. blue) point.

- Any translate $V^{\prime} \in \operatorname{Tr}^{V}$ that intersects $S_{\mathrm{b}, 0}$ (resp. $S_{\mathrm{r}, 0}$ ) contains a blue (resp. red) point, and any translate $W^{\prime} \in \operatorname{Tr}^{W}$ that intersects $S_{\mathrm{b}, 0}$ (resp. $S_{\mathrm{r}, 0}$ ) contains points of both colors.

Color all points of $S_{\mathrm{b}, \mathrm{b}}$ and $S_{\mathrm{b}, 0}$ red, and color all points of $S_{\mathrm{r}, \mathrm{r}}$ and $S_{\mathrm{r}, 0}$ blue. Finally, color $S_{\mathrm{b}, \mathrm{r}}$ using the ANC property of the pair $(V, W)$, and similarly, color $S_{\mathrm{r}, \mathrm{b}}$ also using the ANC property, but the roles of red and blue switched. Now it is easy to check that in this coloring any translate of $V$ or $W$ that contains sufficiently many points of $S$ contains a point of both colors.

Remark In [8] it is proved that if $\{V, W\}$ is a Special pair, then $\{V, W\}$ is not ANC. So, the following statement holds as well.

Lemma $3^{\prime}$ If a pair of wedges is ANC, then it is also NC.

Lemma 4 Let $\mathcal{W}=\{V, W\}$ be a set of two wedges of type 1 (Big). Then $\mathcal{W}$ is $N C$.

Proof By Lemma 3, it is enough to show that $\{V, W\}$ is ANC. Let $W$ be the wedge whose angle is at least $\pi$. Then $W$ is the union of two halfplanes, say, $H_{1}$ and $H_{2}$. Translate both halfplanes so that they contain exactly one point of $S$ and denote them by $X_{1}$ and $X_{2}$, respectively. Note that $X_{1}$ may coincide with $X_{2}$. Color $X_{1}$ and $X_{2}$ red, and all the other points blue. Then any translate of $W$ that contains at least one point contains a red point, and any translate of $V$ that contains at least three points contains a blue point.

Lemma 5 Let $\mathcal{W}=\{V, W\}$ be a set of two wedges of type 2 (Halfplane). Then $\mathcal{W}$ is $N C$.

Proof Again, it is enough to show that they are ANC. Since $\{V, W\}$ is of type 2 (Halfplane), $B d^{V}(S)$ and $B d^{W}(S)$ have at most one point in common. If $B d^{V}(S)$ and $B d^{W}(S)$ are disjoint, then color $B d^{V}(S)$ blue, $B d^{W}(S)$ red, and the other points arbitrarily. Then any nonempty translate of $V$ (resp. $W$ ) contains a blue (resp. red) point. 
Otherwise, let $X$ be their common point. Let $P=B d^{V}(S) \cup B d^{W}(S) \backslash X$, and consider its $V$-boundary $B d^{V}(P)$ and $W$-boundary $B d^{W}(P)$. Clearly, each point in $B d^{V}(S) \backslash X$ belongs to $B d^{V}(P)$, and each point in $B d^{W}(S) \backslash X$ belongs to $B d^{W}(P)$.

If $B d^{V}(P)$ and $B d^{W}(P)$ are disjoint, then color $B d^{V}(S)$ blue, $B d^{W}(P)$ and the other points red. Then any nonempty translate of $V$ contains a blue point. Suppose that we have a translate of $W$ with two points, both blue. Then it should contain $X$ and a point of $B d^{V}(P)$. But this contradicts our assumption that $B d^{V}(P)$ and $B d^{W}(P)$ are disjoint. So, any translate of $W$ which contains at least two points of $S$ contains a red point.

If $B d^{V}(P)$ and $B d^{W}(P)$ are not disjoint, then they have one point in common; let $Y$ be their common point. If $Y$ belongs to $B d^{W}(S)$, then color $B d^{V}(S)$ blue, $B d^{W}(P)$ and the other points red. Then, by the same argument as before, any nonempty translate of $V$ contains a blue point, and any translate of $W$ which contains at least two points of $S$ contains a red point. Finally, if $Y$ belongs to $B d^{V}(P)$, then we proceed analogously, but the roles of $V$ and $W$, and the colors are switched.

Lemma 6 Let $\mathcal{W}=\{V, W\}$ be a set of two wedges of type 4 (Hard). Then $\mathcal{W}$ is $N C$.

Proof As usual, we only prove that $\{V, W\}$ is ANC. Assume that $W$ contains the positive $x$-axis. Just like in the definition of type 4 (Hard), extend the boundary halflines of $W$ to lines. They divide the plane into four parts, Upper, Lower, Left, and Right, which is $W$ itself. We can assume without loss of generality that $V$ contains the negative $x$-axis, one side of $V$ is in Upper, and one side is in Left.

Observe that if a translate of $V$ and a translate of $W$ intersect each other, then one of them contains the apex of the other.

Claim 1 For any point set $P$ and $X \in P$, either $B d^{V}(P \backslash X) \backslash B d^{V}(P)=\emptyset$ or $B d^{W}(P \backslash X) \backslash B d^{W}(P)=\emptyset$.

Proof Suppose on the contrary that $Y \in B d^{V}(P \backslash X) \backslash B d^{V}(P)$ and $Z \in$ $B d^{W}(P \backslash X) \backslash B d^{W}(P)$. Then $X \in V(Y)$ and $X \in W(Z)$, so $V(Y)$ and $W(Z)$ intersect each other; therefore, one of them contains the apex of the other, say, $Z \in V(Y)$. But this is a contradiction, since $Y$ is a boundary point of $P \backslash X$.

Return to the proof of Lemma 6. Color $B d^{V}(S) \backslash B d^{W}(S)$ red, $B d^{W}(S) \backslash B d^{V}(S)$ blue, and the interior points arbitrarily. Now consider the points of $B d^{V}(S) \cap B d^{W}(S)$. For any $X \in B d^{V}(S) \cap B d^{W}(S)$, if $B d^{V}(S \backslash X) \backslash B d^{V}(S) \neq \emptyset$, then color it red, and if $B d^{W}(S \backslash X) \backslash B d^{W}(S) \neq \emptyset$, then color it blue. For each of the remaining points $Y$, we have $B d^{V}(S \backslash Y) \backslash B d^{V}(S)=B d^{W}(S \backslash Y) \backslash B d^{W}(S)=\emptyset$. Color each of these points so that they have the opposite color than the previous point of $B d^{V}(S) \cap B d^{W}(S)$, in the $y$-ordering.

To prove that this coloring is good, let $V^{\prime} \in \operatorname{Tr}_{2}^{V}, V^{\prime} \cap S=\{X, Y\}$. If it intersects $B d^{V}(S) \backslash B d^{W}(S)$, we are done. So assume that $V^{\prime} \cap B d^{V}(S) \subset B d^{V}(S) \cap B d^{W}(S)$. Let $X \in V^{\prime} \cap B d^{V}(S)$. If $X$ is red, then by the coloring rule, $B d^{V}(S \backslash X) \backslash B d^{V}(S)=\emptyset$. However, then $Y$ is also a $V$-boundary point, so we have $Y \in B d^{V}(S) \cap B d^{W}(S)$. 
Again we can assume that $Y$ is red, so $B d^{V}(S \backslash Y) \backslash B d^{V}(S)=\emptyset$. Suppose that $X<_{y} Y$. Since $V^{\prime} \cap S=\{X, Y\}, X$ and $Y$ are consecutive points of $B d^{V}(S) \cap$ $B d^{W}(S)$. Now it is not hard to see that $B d^{W}(S \backslash Y) \backslash B d^{W}(S)=\emptyset$. Therefore, by the coloring rule, $X$ and $Y$ have different colors. For translates of $W$, the argument is analogous, with the colors switched.

Now we turn to the case where we have more than two wedges.

Lemma 7 For any integers $s, t>0$, there is a number $f(s, t)$ with the following property.

Let $\mathcal{W}=\left\{W_{1}, W_{2}, \ldots, W_{t}\right\}$ be a set of $t$ wedges such that any pair $\left\{W_{i}, W_{j}\right\}$ is $N C$, and let $S$ be a set of points. Then $S$ can be decomposed into $t$ parts $S_{1}, S_{2}, \ldots, S_{t}$ such that for $i=1,2, \ldots, t$ and for any translate $W_{i}^{\prime}$ of $W_{i}$, if $\left|W_{i}^{\prime} \cap S\right| \geq f(s, t)$, then $\left|W_{i}^{\prime} \cap S_{i}\right| \geq s$.

Proof The existence of $f(1,2)$ is equivalent to the property that the corresponding two wedges are ANC. Now we show that $f(s, 2)$ exists for every $s$. Let $V$ and $W$ be two wedges that form an NC pair. Let $P_{1}^{V}, P_{2}^{V}, \ldots, P_{s^{2} f(1,2)}^{V}$ be the path decomposition of $S$ of order $s^{2} f(1,2)$ with respect to $V$. For $i=1,2, \ldots, s$, let

$$
H_{i}=\bigcup_{j=(i-1) s f(1,2)+1}^{i s f(1,2)} P_{j}^{V} .
$$

For each $H_{i}$, take the $W$-path decomposition, $P_{1}^{W}\left(H_{i}\right), \ldots, P_{s f(1,2)}^{W}\left(H_{i}\right)$, and for $j=$ $1,2, \ldots, s$, let

$$
H_{i}^{j}=\bigcup_{k=(j-1) f(1,2)+1}^{j f(1,2)} P_{k}^{W}\left(H_{i}\right) .
$$

For every $i, j=1,2, \ldots, s$, color $H_{i}^{j}$ so that any translate of $V$ (resp. $W$ ) that intersects it in at least $f(1,2)$ points contains at least one red (resp. blue) point of it. This is possible, since the pair $\{V, W\}$ is ANC.

Consider a translate $V^{\prime}$ of $V$ that contains at least $s^{2} f(1,2)$ points of $S$. For every $i, V^{\prime}$ intersects $H_{i}$ in $s f(1,2)$ points, so there is $j$ such that it intersects $H_{i}^{j}$ in at least $f(1,2)$ points. Therefore, $V^{\prime}$ contains at least one red point of $H_{i}^{j}$ and thus at least $s$ red points of $S$.

Consider now a translate $W^{\prime}$ of $W$ that contains at least $s^{2} f(1,2)$ points of $S$. There is $i$ such that $W^{\prime}$ intersects $H_{i}$ in at least $s f(1,2)$ points. Therefore, it intersects each of $P_{1}^{W}\left(H_{i}\right), \ldots, P_{s f(1,2)}^{W}\left(H_{i}\right)$, in at least one point, so for $j=1,2, \ldots, s, W^{\prime}$ intersects $H_{i}^{j}$ in at least $f(1,2)$ points. Consequently, it contains at least one blue point of each $H_{i}^{j}$ and thus at least $s$ blue points of $S$.

Now let $s, t>2$ fixed and suppose that $f\left(s^{\prime}, t-1\right)$ exists for every $s^{\prime}$. Let $\left\{W_{1}, W_{2}, \ldots, W_{t}\right\}$ be our set of wedges such that any pair of them is NC. Let $s^{\prime}=$ $f(s, 2)$. Partition our point set $S$ into $S_{1}^{\prime}, S_{2}^{\prime}, \ldots, S_{t-1}^{\prime}$ such that for $i=1,2, \ldots, t-1$ and for any translate $W_{i}^{\prime}$ of $W_{i}$, if $\left|W_{i}^{\prime} \cap S\right| \geq f\left(s^{\prime}, t-1\right)$, then $\left|W_{i}^{\prime} \cap S_{i}^{\prime}\right| \geq s^{\prime}=$ 
$f(s, 2)$. For each $i=1,2, \ldots, t-1$, partition $S_{i}^{\prime}$ into two parts, $S_{i}^{\prime \prime}$ and $S_{i}^{t}$, such that for any translate $W_{i}^{\prime}$ of $W_{i}$, if $\left|W_{i}^{\prime} \cap S_{i}^{\prime}\right| \geq f(s, 2)$, then $\left|W_{i}^{\prime} \cap S_{i}^{\prime \prime}\right| \geq s$, and for any translate $W_{t}^{\prime}$ of $W_{t}$, if $\left|W_{t}^{\prime} \cap S_{i}^{\prime}\right| \geq f(s, 2)$, then $\left|W_{t}^{\prime} \cap S_{i}^{t}\right| \geq s$. Finally, for $i=1,2, \ldots, t-1$, let $S_{i}=S_{i}^{\prime \prime}$, and let $S_{t}=\bigcup_{j=1}^{t-1} S_{j}^{t}$. For $i=1,2, \ldots, t-1$ and any translate $W_{i}^{\prime}$ of $W_{i}$, if $\left|W_{i}^{\prime} \cap S\right| \geq f\left(s^{\prime}, t-1\right)$, then $\left|W_{i}^{\prime} \cap S_{i}^{\prime}\right| \geq s^{\prime}=f(s, 2)$, so $\left|W_{i}^{\prime} \cap S_{i}\right| \geq s$,

Moreover, for any translate $W_{t}^{\prime}$ of $W_{t}$, if $\left|W_{i}^{\prime} \cap S\right| \geq f\left(s^{\prime}, t-1\right)$, then for some $i=1,2, \ldots t-1,\left|W_{t}^{\prime} \cap S_{i}^{\prime}\right| \geq \frac{f\left(s^{\prime}, t-1\right)}{t-1} \geq f(s, 2)$; therefore, $\left|W_{t}^{\prime} \cap S_{i}^{t}\right| \geq s$, so $\left|W_{t}^{\prime} \cap S_{t}\right| \geq s$. This concludes the proof of Lemma 7 .

Remark The proofs of Lemmas $2,4,5$, and 6 imply that $f(1,2) \leq 8$. Combining it with the proof of Lemma 7 , we get the bound $f(s, t) \leq(8 s)^{2^{t-1}}$.

Lemma 8 A set of wedges $\mathcal{W}=\left\{W_{1}, W_{2}, \ldots, W_{t}\right\}$ is $N C$ if and only if any pair $\left\{W_{i}, W_{j}\right\}$ is $N C$.

Proof Clearly, if some pair $\left\{W_{i}, W_{j}\right\}$ is not NC, then the whole set $\mathcal{W}$ is not NC either. Suppose that every pair $\left\{W_{i}, W_{j}\right\}$ is NC. Decompose $S$ into $t$ parts $S_{1}, S_{2}, \ldots, S_{t}$ with the property that for $i=1,2, \ldots, t$ and for any translate $W_{i}^{\prime}$ of $W_{i}$, if $\left|W_{i}^{\prime} \cap S\right| \geq$ $f(3, t)$, then $\left|W_{i}^{\prime} \cap S_{i}\right| \geq 3$. Then, by Lemma 1, each $S_{i}$ can be colored with red and blue so that if $\left|W_{i}^{\prime} \cap S_{i}\right| \geq 3$, then $W_{i}^{\prime}$ contains points of both colors. So this coloring of $S$ has the property that for $i=1,2, \ldots, t$ and for any translate $W_{i}^{\prime}$ of $W_{i}$, if $\left|W_{i}^{\prime} \cap S\right| \geq f(3, t)$, then it contains points of both colors.

\section{Proof of Theorem 1}

Suppose that $P$ is an open convex polygon of $n$ vertices and $\mathcal{P}=\left\{P_{i} \mid i \in I\right\}$ is a collection of translates of $P$ which forms an $M$-fold covering of the plane. We will set the value of $M$ later. Let $m$ be the minimum distance between any vertex and nonadjacent side of $P$. Take a square grid $\mathcal{G}$ of basic distance $m / 2$. Obviously, any translate of $P$ intersects at most $K=4 \pi(\operatorname{diam}(P)+m)^{2} / m^{2}$ basic squares. For each (closed) basic square $B$, using its compactness, we can find a finite subcollection of the translates such that they still form an $M$-fold covering of $B$. Take the union of all these subcollections. We have a locally finite $M$-fold covering of the plane. That is, every compact set is intersected by finitely many of the translates. It is sufficient to decompose this covering. For simplicity, use the same notation $\mathcal{P}=\left\{P_{i} \mid i \in I\right\}$ for this subcollection.

We formulate and solve the problem in its dual form. Let $O_{i}$ be the center of gravity of $P_{i}$. Since $\mathcal{P}$ is an $M$-fold covering of the plane, every translate of $\bar{P}$, the reflection of $P$ through the origin, contains at least $M$ points of the locally finite set $\mathcal{O}=\left\{O_{i} \mid i \in I\right\}$.

The collection $\mathcal{P}=\left\{P_{i} \mid i \in I\right\}$ can be decomposed into two coverings if and only if the set $\mathcal{O}=\left\{O_{i} \mid i \in I\right\}$ can be colored with two colors so that every translate of $\bar{P}$ contains a point of both colors. 
Let $\mathcal{W}=\left\{W_{1}, W_{2}, \ldots, W_{n}\right\}$ be the set of wedges that correspond to the vertices of $\bar{P}$. By the convexity of $\bar{P}$, no pair $\left\{W_{i}, W_{j}\right\}$ is of type 5 (Special); therefore, by the previous lemmas, each pair is NC. Consequently, by Lemma $8, \mathcal{W}$ is NC as well. So there is a $k$ with the following property:

* Any set of points $S$ can be colored with two colors so that any translate of $W_{1}, W_{2}, \ldots, W_{n}$ that contains at least $k$ points of $S$ contains points of both colors.

Choose $M$ such that $M \geq k K$, and color the points of $\mathcal{O}$ in each basic square separately with property *.

Since any translate $P^{\prime}$ of $\bar{P}$ intersects at most $K$ basic squares of the grid $\mathcal{G}, P^{\prime}$ contains at least $M / K \geq k$ points of $\mathcal{O}$ in the same basic square $B^{\prime}$. By the choice of the grid $\mathcal{G}, B^{\prime}$ contains at most one vertex of $P^{\prime}$, and hence $B^{\prime} \cap P^{\prime}=B^{\prime} \cap W$, where $W$ is a translate of some $W_{i} \in \mathcal{W}$. So, by property *, $P^{\prime}$ contains points of $\mathcal{O} \cap B^{\prime}$ of both colors. This concludes the proof of Theorem 1 .

\section{Concluding Remarks}

Throughout this paper we made no attempt to optimize the constants. However, it may be an interesting problem to determine (asymptotically) the smallest $k$ in the proof of Theorem 1.

Another interesting question is to decide whether this constant depends only on the number of vertices of the polygon or on the shape as well. In particular, we cannot verify the following:

Conjecture There is a constant $k$ such that any $k$-fold covering of the plane with translates of a convex quadrilateral can be decomposed into two coverings.

One can also investigate whether a given covering can be decomposed into $s$ coverings for some fixed $s$. For any planar set $P$ and $s \geq 1$, if it exists, let $k=k(s, P)$ be the smallest number such that any $k$-fold covering of the plane with translates of $P$ can be decomposed into $s$ coverings. By Theorem $1, k(2, P)$ exists for any open convex polygon $P$. With a slight modification of its proof, we get the following more general result.

Theorem 1' For any open convex polygon $P$ and any $s$, there exists a (smallest) number $k=k(s, P)$ such that any $k$-fold covering of the plane with translates of $P$ can be decomposed into s coverings.

Our proof gives $k(s, P)<K_{P}(8 s)^{2^{n-1}}$, where $K_{P}$ is the constant $K$ from the proof of Theorem 1 , and $n$ is the number of vertices of $P$. For centrally symmetric open convex polygons, Pach and Tóth [6] established a much better upper bound, which is quadratic in $s$. Recently, Aloupis et al. [1] improved it to linear one. They showed that, for any centrally symmetric open convex polygon $P$, there is $\alpha_{P}$ such that $k(s, P)<$ $\alpha_{P} s$. The best known lower bound on $k(s, P)$ is $(s, P) \geq\lfloor 4 s / 3\rfloor-1[6]$. 
Our proofs use the assumption that the covering is locally finite, and for open polygons, we could find a locally finite subcollection which is still a $k$-fold covering. Still, we strongly believe that Theorem 1 holds for closed convex polygons as well.

Acknowledgement We are very grateful to the anonymous referee for his remarks.

\section{References}

1. Aloupis, G., Cardinal, J., Collette, S., Langerman, S., Orden, D., Ramos, P.: Decomposition of multiple coverings into more parts. In preparation, arXiv:0807.0552v1

2. Brass, P., Pach, J., Moser, W.: Research Problems in Discrete Geometry. Springer, Berlin (2005)

3. Mani-Levitska, P., Pach, J.: Decomposition problems for multiple coverings with unit balls. Manuscript (1986)

4. Pach, J.: Decomposition of multiple packing and covering. Kolloq. Math. Inst. Univ. Salzburg. Diskrete Geom. 2, 169-178 (1980)

5. Pach, J.: Covering the plane with convex polygons. Discrete Comput. Geom. 1, 73-81 (1986)

6. Pach, J., Tóth, G.: Decomposition of multiple coverings into many parts. In: Proc. of the 23rd ACM Symposium on Computational Geometry (SoCG07) 2007, pp. 133-137. Also in: Comput. Geom. Theory Appl. 42, 127-133 (2009)

7. Pach, J., Tardos, G., Tóth, G.: Indecomposable coverings. In: The China-Japan Joint Conference on Discrete Geometry, Combinatorics and Graph Theory (CJCDGCGT 2005). Lecture Notes in Computer Science, vol. 4381, Springer, Berlin (2007)

8. Pálvölgyi, D.: Indecomposable coverings with concave polygons. In preparation

9. Tardos, G., Tóth, G.: Multiple coverings of the plane with triangles. Discrete Comput. Geom. 38, 443450 (2007) 